\title{
Serum RDW Level of COPD Patients and the Characteristics of the Exacerbation

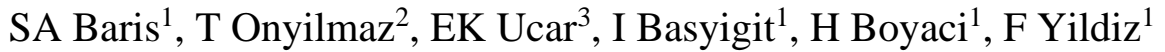

\begin{abstract}
Affiliations:
${ }^{1}$ Kocaeli University School of Medicine, Department of Pulmonary Diseases, Kocaeli, Turkey ${ }^{2}$ Private Konak Hospital, Department of Pulmonary Diseases, Kocaeli, Turkey ${ }^{3}$ Tekirdag Çorlu Government Hospital, Department of Pulmonary Diseases, Tekirdag, Turkey
\end{abstract}

\section{Correspondence:}

Dr S Baris

Department of Pulmonary Diseases

Kocaeli University School of Medicine

Umuttepe

41380, Kocaeli

Turkey

E-mail:serapargun2002@yahoo.com

Short title: COPD and RDW Level

Synopsis: Red blood cell distribution width (RDW) is a measure of hemogram. Nearly one third of COPD patients have increased RDW levels and exitus rates are higher in this group. It is suggested that elevated RDW might be related with increased mortality in COPD patients. 


\section{ABSTRACT}

Objectives: Red blood cell distribution width (RDW) is a measure of red blood cell size heterogeneity. The aim of this study was to evaluate the relationship between the RDW level and characteristics of the COPD exacerbations.

Methods: The records of the hospitalized COPD patients were evaluated retrospectively. The demographic characteristics, duration of follow-up and the numbers of exacerbations and hospitalizations, duration of the hospital stay, need of non-invasive mechanic ventilation (NIMV) or intensive care unit (ICU) and laboratory findings were recorded.

Results: There were 35 women (18.5\%), 154 men (81.5\%), totally 189 patients whose mean age was $67.4 \pm 10.6$ years. The mean durations of follow-up and hospitalization were respectively $2.85 \pm 2.5$ years and $8.5 \pm 5.4$ days. Forty one of the patients $(22 \%)$ were current smoker and 115 of them $(61.8 \%)$ were ex-smoker. Nearly one third of the patients $(34.9 \%)$ had increased RDW levels. When the patients with increased RDW level compared to normal ones; no statistically significant difference was found with respect to the gender, smoking history, smoking package of years, numbers of the exacerbation and hospitalization, duration of hospital stay and need of NIMV or ICU. However, it was found that the presence of exitus was significantly higher in patients with increased RDW level $(\mathrm{p}=0.02)$.

Conclusion: This study suggested that increased level of RDW might be related with increased mortality in COPD patients. Further long-term prospective studies with healthy controls and COPD patients in both stable or exacerbation period are needed in order to evaluate clinical significance of these findings.

Keywords: COPD, RDW, exacerbation, hospitalization, mortality 


\section{INTRODUCTION}

Red blood cell distribution width (RDW) is a measure of red blood cell size heterogeneity and it reflects the erythrocyte morphology (1). It is a component of complete blood count and it is used in the differential diagnosis of anemia (2). Furthermore; systemic inflammation, ineffective erythropoiesis, nutritional deficiencies, bone marrow dysfunction or increased destruction can also cause a higher RDW. Recent evidences report that acute or chronic disorders such as cardiovascular diseases (CVD), cancer, sepsis, chronic obstructive pulmonary disease (COPD), pulmonary thromboembolism (PTE), diabetes mellitus, liver and kidney failure are related with increased RDW levels (1-4).

It is also reported that higher RDW levels may reflect underlying chronic inflammation which can result in an increased mortality (3). There is an association between increased RDW levels and high mortality risk in both general population and some diseases including cardiovascular diseases and COPD (2, 3). COPD is a systemic disease and inflammation has an important role on development of COPD. COPD-related inflammation may also impair erythropoiesis and cause increased RDW levels, as do other chronic inflammatory processes. But, there are limited studies evaluating the relation among COPD, mortality and RDW levels $(1,3)$.

COPD is treatable and preventable systemic disease characterized by persistent airflow limitation that is usually progressive and associated with an increased inflammatory response to noxious particles and gases (5). It is fifth leading cause of death and estimated to be the third in 2030 worldwide (6). Exacerbations are an important cause of mortality and morbidity in COPD patients $(7,8)$. The aim of this study is to evaluate the relationship between the RDW level and characteristics of the COPD exacerbations. 


\section{METHODS}

The medical records of the hospitalized patients with COPD exacerbation in Pulmonary Diseases Clinic were evaluated retrospectively. The exacerbation of the COPD was defined as an acute event characterized by a worsening of the respiratory symptoms that was beyond normal day-to-day variations and led to a change in medication (5).

The demographic characteristics, smoking history, duration of follow-up and the numbers of exacerbations and hospitalizations, duration of the hospital stay, need of noninvasive mechanic ventilation (NIMV) or intensive care unit (ICU) were recorded. Laboratory findings including hemogram and artery blood gas analysis which were taken on first day of hospitalization were also recorded.

\section{Exclusion criteria}

Patients with other lung diseases such as tuberculosis, bronchiectasis and interstitial lung disease were excluded. Patients with iron or vitamin deficiencies (such as B12 or folate) and recent transfusions were also excluded from the study.

\section{Laboratory analysis}

Red cell distribution width was assessed by flow cytometric analyzers as the part of the routine full blood count. RDW was calculated as (standard deviation of red cell volume/mean cell volume $) \times 100$. The normal range was between $12.2 \%$ and $17.2 \%$ in our laboratory.

\section{Statistical analysis}

Statistical analyses of the study were performed using IBM SPSS for Windows version 20.0 (SPSS, Chicago, IL, USA). Categorical variables were expressed as counts (percentage). Numeric variables were expressed as mean and standard deviation. Comparisons of categorical variables between the groups were performed using the Chi square test. A twosided $P$ value $<0.05$ was considered statistically significant. 


\section{RESULTS}

There were 35 women $(18.5 \%), 154$ men $(81.5 \%)$, totally 189 patients. The mean age was $67.4 \pm 10.6$ years. The mean durations of follow-up and hospitalization were respectively $2.85 \pm 2.5$ years and $8.5 \pm 5.4$ days. Forty one of the patients $(22 \%)$ were current smoker and 115 of them $(61.8 \%)$ were ex-smoker. Respiratory infections $(72 \%)$ were the most common causes of the exacerbations. Demographic data, the characteristics of exacerbation and laboratory findings of the patients were shown in Table-1 and Table-2.

It was found that level of RDW was increased nearly one third of the patients (34.9\%). Retrospective analyses of 189 COPD patients revealed that the RDW level had a mean value of $16.95 \pm 2.2$. The comparison of exacerbation characteristics and laboratory parameters according to RDW levels were shown in Table-3.

When the patients with increased RDW level compared to normal ones; no statistically significant difference was found with respect to the gender, smoking history, smoking package of years, numbers of the exacerbation and hospitalization, duration of hospital stay and need of NIMV or ICU (Table-4). However, it was found that the presence of exitus was significantly higher in patients with increased RDW level $(\mathrm{p}=0.02)$ (Figure-1).

\section{DISCUSSION}

This study demonstrated that nearly one third of the hospitalized COPD patients with exacerbation had increased RDW levels. There were no statistically significant differences of gender, smoking history, smoking package of years, numbers of the exacerbation and hospitalization, duration of hospital stay and need of NIMV or ICU in patients with increased RDW level compared to normal ones. However, it was found that the presence of exitus was significantly higher in patients with increased RDW level. 
The red blood cell distribution width (RDW) is a simple and cheap parameter, which is a component of hemogram. It reflects the degree of heterogeneity of erythrocyte volume. It is also known as anisocytosis. RDW is used for differential diagnosis of anemias (2). Beside this, RDW is a recently recognized biomarker of adverse outcome in various acute and chronic conditions (9). An increased RDW is related with both impaired erythropoiesis and abnormal red blood cell survival, which may be attributed to a variety of underlying metabolic abnormalities such as oxidative stress, inflammation, erythrocyte fragmentation and alteration of erythropoietin function (2).

Anisocytosis can also seen in some disorders such as cardiovascular disease, cancer, sepsis, diabetes, liver/kidney failure and respiratory diseases including community acquired pneumonia (CAP), venous thromboembolism and COPD (2,4,10-12). Studies evaluating the relation between COPD and RDW levels are limited $(1,3)$. In a retrospective analysis of stable COPD patients, it is revealed that RDW is positively correlated with C-reactive protein (CRP), right ventricular dysfunction and pulmonary arterial hypertension (3). In a multivariable logistic regression analysis, the presence of high RDW is found to be only independent parameter predicting RV failure in COPD patients and it is suggested that RDW may be used to identify COPD patients with RV failure (13).

COPD is treatable and preventable systemic disease characterized by persistent airflow limitation that is usually progressive (5). It is one of the most important causes of mortality and morbidity worldwide. Particularly in older age groups in the United States, it is among the 10 most important disease causing hospitalizations and death with increasing prevalence day by day (14). Exacerbations are most frequent cause of morbidity, hospital admission and mortality in COPD (15). The exacerbation of the COPD is defined as an acute event characterized by worsening of the respiratory symptoms that was beyond normal day-to-day variations and led to a change in medication (5). Respiratory failure can be seen in 
exacerbation of COPD. The arterial blood gas analysis is an important tool for defining respiratory failure and to start NIMV therapy in COPD patients. The relation between RDW and arterial blood gas analysis parameters of COPD patients are also investigated. It is reported that oxygen saturation is significantly lower for patients with an elevated RDW (1). In our study, it was also found that mean $\mathrm{Pa} \mathrm{O}_{2}$ and oxygen saturation values were lower in patients with increased RDW levels compared to normal ones. But, the difference was not statistically significant. Beside this, mean $\mathrm{PaCO}_{2}$ was significantly higher in patients with increased RDW level compared to normal ones $(51.03 \pm 15.1$ vs. $47.2 \pm 13.6, p=0.007)$. It is suggested that RDW level may be useful for predicting hypercapnic COPD patients who needs NIMV therapy.

The stage of COPD is assessed by pulmonary function test, health status and presence of exacerbation / hospitalization (5). It reflects the severity of disease. There is a study evaluating the relation between RDW level and the stage of disease. The highest RDW is observed in the very severe stage $(p<0.001)$ of COPD patients in Tertemiz et al.'s study $(1)$. In our study, it was not possible to compare the RDW levels according to stages of disease due to its retrospective design. There was a lack of data about health status assessed by modified Medical Research Council (mMRC) Questionnaire or COPD assessment test (CAT) due to retrospective nature of this study. Furthermore, most of the patients were not able to perform the pulmonary function test since they had a severe dyspnea due to exacerbation.

The association between RDW level and length of stay (LOS) in hospital is also investigated in recent studies $(11,16)$. RDW is associated with length of stay in hospital and use of vasopressors in hospitalized patients with CAP (11). Greater initial RDW and change of RDW during heart failure hospitalization are associated with longer LOS, suggesting both initial RDW and change of RDW during hospitalization may be helpful in personalizing prognosis and treatment (16). In contrast, there was no significant difference of LOS in 
hospital between the groups in our study. It is thought that study populations including different diseases might be responsible for opposite results.

The baseline RDW and increase in RDW during hospitalization are also compared in patients with sepsis and heart failure $(16,17)$. It is reported that there is an increase in RDW from baseline during the first 72 hours after hospitalization and it is significantly associated with adverse clinical outcomes in sepsis. Therefore, it is suggested that a combination of baseline RDW value and an increase in RDW can be a promising independent prognostic marker in patients with severe sepsis or septic shock (17). It is also suggested that both initial RDW and change of RDW during hospitalization may be helpful in personalizing prognosis and treatment in patients with heart failure (16). However, it was not possible to evaluate the role of RDW changes during the hospitalization on adverse clinical outcomes due to retrospective design of this study.

A potential independent association is recently demonstrated between high RDW levels and the risk of all-cause mortality in critically ill patients, although the underlying mechanism is still unclear (17). Elevated RDW has been associated with adverse outcomes of heart failure, infective endocarditis, VTE, and idiopathic pulmonary hypertension $(9,18)$. RDW is recognized as a mortality marker in patients with CVD (3). It is also reported that baseline RDW is significantly associated with incidence of fatal cardiac events (19). An increased RDW conveys important information for both short and long term prognosis (3). Greater initial RDW and change of RDW during heart failure hospitalization are associated with 30-day mortality (16). RDW is found to be associated with 30-day mortality in hospitalized patients with CAP (11). Even more importantly, the value of RDW is now being regarded as a strong and independent risk factor for death in the general population (2). Furthermore, studies evaluating the role of RDW on mortality in COPD patients are also support these findings $(1,3)$. Elevated RDW levels are associated with increased mortality 
risk in stable COPD patients (3). It is suggested that there is a correlation between survival of COPD patients and RDW levels (1). Also, the presence of exitus was significantly higher in patients with increased RDW level in our study as similar to literature.

There were several limitations of this study that should be considered. First, it was a retrospective investigation, and data collection was based on medical records. Second, it reflected the RDW levels of only hospitalized patients with COPD exacerbation. So the study group did not include outpatients and study population was relatively small. Furthermore, it was not possible to compare the RDW results of the patients in stable or exacerbation periods. Third, only baseline RDW levels of the patient were recorded, so comparing the role of changes of RDW levels during the hospitalization on adverse clinical outcomes was not possible. Forth, it was not possible to assess the patient's health status by modified Medical Research Council (mMRC) Questionnaire or COPD assessment test (CAT) and evaluate the new classification of COPD because of its retrospective nature. So, the severity of the disease was not classified.

\section{CONCLUSION}

Early detection and effective treatment of exacerbations are key factors that may reduce in hospital admissions. RDW is a simple, easy to perform and cheap parameter. It might be useful for predicting hypercapnic COPD patients who needs NIMV therapy in exacerbation period. Also it is suggested that increased level of RDW might be related with increased mortality in COPD patients. Further long-term prospective studies with healthy controls and COPD patients in both stable or exacerbation period are needed in order to evaluate clinical significance of these findings. 


\section{AUTHORS' NOTE}

S Argun Baris conceived paper, oversaw data collection, conducted data analysis, wrote manuscript and approved final version. T Onyilmaz participated in study design, data collection, wrote manuscript and approved final version. E Kuslu Ucar participated in study design, data collection, and revision of manuscript and approved final version. I Basyigit participated in study design, interpretation of data and revision of manuscript and approved final version. $\mathrm{H}$ Boyaci participated in study design and interpretation of data; critically revised manuscript and approved final version. F Yildiz participated in study design and interpretation of data, critically revised manuscript and approved final version. The authors declare that they have no conflicts of interest. 


\section{REFERENCES}

1. Tertemiz KC, Ozgen Alpaydin A, Sevinc C, Ellidokuz H, Acara AC, Cimrin A. Could "red cell distribution width" predict COPD severity? Rev Port Pneumol 2016 Jan 22. pii: S2173-5115(15)00210-9. doi: 10.1016/j.rppnen.2015.11.006. [Epub ahead of print]

2. Salvagno GL, Sanchis-Gomar F, Picanza A, Lippi G. Red blood cell distribution width: A simple parameter with multiple clinical applications. Crit Rev Clin Lab Sci. 2015; 52(2):86-105. doi: 10.3109/10408363.2014.992064.

3. Seyhan EC, Özgül MA, Tutar N, Ömür I, Uysal A, Altin S. Red blood cell distribution and survival in patients with chronic obstructive pulmonary disease. COPD 2013; 10(4):416-24. doi: 10.3109/15412555.2012.758697.

4. Zöller B, Melander O, Svensson P, Engström G. Red cell distribution width and risk for venous thromboembolism: a population-based cohort study. Thromb Res 2014; 133(3):334-9. doi: 10.1016/j.thromres.2013.12.013.

5. Global Initiative for Chronic Obstructive Lung Disease (GOLD). Global strategy for the diagnosis, management, and prevention of chronic obstructive pulmonary disease: updated 2014. http://www.goldcopd.org/uploads/users/files/GOLD_Report2014 Feb07

6. http://www.who.int/respiratory/copd/burden/en/ [accessed 06.07.14].

Celli BR, Mac Nee W. ATS/ERS Task Force. Standards for the diagnosis and care of patients with COPD: a summary of the ATS/ERS position paper. Eur Respir J. 2004; 23: 932-46.

7. Miravitlles M, Izquierdo I, Herrejón A, Torres JV, Baró E, Borja J; ESFERA investigators. COPD severity score as a predictor of failure in exacerbations of COPD. The ESFERA study. Respir Med 2011; 105(5):740-7. 
8. Guray Y, Ipek EG, Guray U, Demirkan B, Kafes H, Asarcikli LD et al. Red cell distribution width predicts mortality in infective endocarditis. Arch Cardiovasc Dis. 2014; 107(5):299-307. doi: 10.1016/j.acvd.2014.04.008.

9. Abul Y, Ozsu S, Korkmaz A, Bulbul Y, Orem A, Ozlu T. Red cell distribution width: a new predictor for chronic thromboembolic pulmonary hypertension after pulmonary embolism. Chron Respir Dis. 2014; 11(2):73-81. doi: 10.1177/1479972314525057.

10. Lee JH, Chung HJ, Kim K, Jo YH, Rhee JE, Kim YJ, Kang KW.Red cell distribution width as a prognostic marker in patients with community-acquired pneumonia. Am $\mathbf{J}$ Emerg Med. 2013;31(1):72-9. doi: 10.1016/j.ajem.2012.06.004.

11. Koma Y, Onishi A, Matsuoka H, Oda N, Yokota N, Matsumoto Y et al. Increased red blood cell distribution width associates with cancer stage and prognosis in patients with lung cancer. PLoS One. 2013; 8(11):e80240. doi: 10.1371/journal.pone.0080240. eCollection 2013.

12. Sincer I, Zorlu A, Yilmaz MB, Dogan OT, Ege MR, Amioglu G et al. Relationship between red cell distribution width and right ventricular dysfunction in patients with chronic obstructive pulmonary disease. Heart Lung. 2012;41(3):238-43. doi: 10.1016/j.hrtlng.2011.07.011.

13. Mannino DM. COPD. Epidemiology, prevalence, morbidity and mortality, and disease heterogenity. Chest. 2002; 121:121S-126S.

14. Miravitlles M, Guerrero T, Mayordomo C, Sánchez-Agudo L, Nicolau F, Segú JL: Factors Associated with Increased Risk of Exacerbation and Hospital Admission in a Cohort of Ambulatory COPD Patients: A Multiple Logistic Regression Analysis. Respiration 2000; 67(5):495-501.

15. Muhlestein JB, Lappe DL, Anderson JL, Muhlestein JB, Budge D, May HT et al. Both initial red cell distribution width (RDW) and change in RDW during heart failure 
hospitalization are associated with length of hospital stay and 30-day outcomes. Int $\mathbf{J}$ Lab Hematol. 2016;38(3):328-37. doi: 10.1111/ijlh.12490.

16. Kim CH, Park JT, Kim EJ, Han JH, Han JS, Choi JY et al. An increase in red blood cell distribution width from baseline predicts mortality in patients with severe sepsis or septic shock. Crit Care. 2013; 17(6):R282. doi: 10.1186/cc13145.

17. Ellingsen TS, Lappegård J, Skjelbakken T, Brækkan SK, Hansen JB. Red cell distribution width is associated with incident venous thromboembolism (VTE) and case-fatality after VTE in a general population. Thromb Haemost. 2015; 113(1):193200. doi: 10.1160/TH14-04-0335.

18. Borné Y, Smith JG, Melander O, Engström G. Red cell distribution width in relation to incidence of coronary events and case fatality rates: a population-based cohort study. Heart. 2014; 100(14):1119-24. doi: 10.1136/heartjnl-2013-305028. 
Table 1: Demographic characteristics of the patients

\begin{tabular}{llll}
\hline & & $\mathbf{n}$ & $\mathbf{\%}$ \\
\hline Gender & Female & 35 & 18.5 \\
& Male & 154 & 81.5 \\
Smoking history & Non smoker & 30 & 16.1 \\
& Current smoker & 41 & 22 \\
& Ex-smoker & 115 & 61.8 \\
RDW & Normal RDW & 123 & 65.1 \\
& Increased RDW & 66 & 34.9 \\
NIMV & $(-)$ & 114 & 60.3 \\
& $(+)$ & 75 & 39.7 \\
ICU & $(-)$ & 175 & 92.6 \\
& $(+)$ & 14 & 7.4 \\
Exitus & $(-)$ & 177 & 93.7 \\
& $(+)$ & 12 & 6.3 \\
\hline
\end{tabular}

Table 2: The characteristics of exacerbation and laboratory parameters

\begin{tabular}{lccc}
\hline & Min & Max & Mean \pm SD \\
\hline Age, years & 35 & 93 & $67.4 \pm 10.6$ \\
Smoking pack/year & 0 & 140 & $42.8 \pm 29.9$ \\
The number of exacerbation , n & 1 & 20 & $2.9 \pm 3.4$ \\
The number of hospitalization, $\mathbf{n}$ & 1 & 12 & $2.1 \pm 2.1$ \\
Duration of hospitalization, day & 1 & 34 & $8.5 \pm 5.4$ \\
Hemoglobin g/dl & 8.9 & 18.1 & $13.03 \pm 1.7$ \\
Hematocrit, \% & 25.2 & 55.1 & $39.8 \pm 5.3$ \\
RDW & 13.4 & 27.1 & $16.95 \pm 2.2$ \\
CRP, mg/dl & 0.04 & 47.82 & $8.04 \pm 10.1$ \\
PaCO, mmHg & 22 & 92 & $48.7 \pm 14.2$ \\
SO,$\%$ & 65.6 & 99.1 & $90.1 \pm 10.1$ \\
\hline
\end{tabular}


Table 3: The comparison of exacerbation characteristics and laboratory parameters according to RDW levels

\begin{tabular}{lccc}
\hline & Normal RDW & Increased RDW & p \\
\hline Age, years & $66.8 \pm 10.8$ & $68.6 \pm 9.97$ & 0.15 \\
Smoking pack/year & $40.8 \pm 28.6$ & $46.3 \pm 32$ & 0.7 \\
The number of exacerbation, $\mathbf{n}$ & $2.9 \pm 3.4$ & $3.02 \pm 3.4$ & 0.1 \\
The number of hospitalization, $\mathbf{n}$ & $2.05 \pm 1.9$ & $2.2 \pm 2.3$ & 0.7 \\
Duration of hospitalization, day & $8.61 \pm 5.8$ & $8.4 \pm 4.5$ & 0.8 \\
$\mathbf{C R P}, \mathbf{m g} / \mathbf{L}$ & $8.6 \pm 10.7$ & $7.02 \pm 8.9$ & 0.42 \\
PaO $_{2}, \mathbf{m m H g}$ & $67.7 \pm 18.9$ & $66.3 \pm 16.7$ & 0.08 \\
$\mathbf{P a C O}_{2}, \mathbf{m m H g}$ & $47.2 \pm 13.6$ & $51.03 \pm 15.1$ & $\mathbf{0 . 0 0 7}$ \\
$\mathbf{S O}_{2}, \boldsymbol{\%}$ & $90.7 \pm 7.8$ & $89.3 \pm 7.6$ & 0.47 \\
\hline
\end{tabular}

Table 4: Demographic characteristics of the patients according to RDW level

\begin{tabular}{llccccc}
\hline & & \multicolumn{2}{c}{ Normal RDW } & \multicolumn{2}{c}{ Increased RDW } & \\
& & $\mathbf{n}$ & $\mathbf{\%}$ & $\mathbf{n}$ & $\mathbf{\%}$ & $\mathbf{p}$ \\
\hline Gender & Female & 19 & 15.4 & 16 & 24.2 & 0.13 \\
& Male & 104 & 84.6 & 50 & 75.8 & \\
history & Non smoker & 19 & 15.8 & 11 & 16.7 & \\
& Current smoker & 25 & 20.8 & 16 & 24.2 & 0.8 \\
& Ex smoker & 76 & 63.3 & 39 & 59.1 & \\
NIMV & $(-)$ & 80 & 65 & 34 & 51.5 & 0.07 \\
& $(+)$ & 43 & 35 & 32 & 48.5 & \\
ICU & $(-)$ & 116 & 94.3 & 59 & 89.4 & 0.07 \\
& $(+)$ & 7 & 5.7 & 7 & 10.6 & \\
Exitus & $(-)$ & 119 & 96.7 & 58 & 87.9 & $\mathbf{0 . 0 2}$ \\
& $(+)$ & 4 & 3.3 & 8 & 12.1 & \\
\hline
\end{tabular}




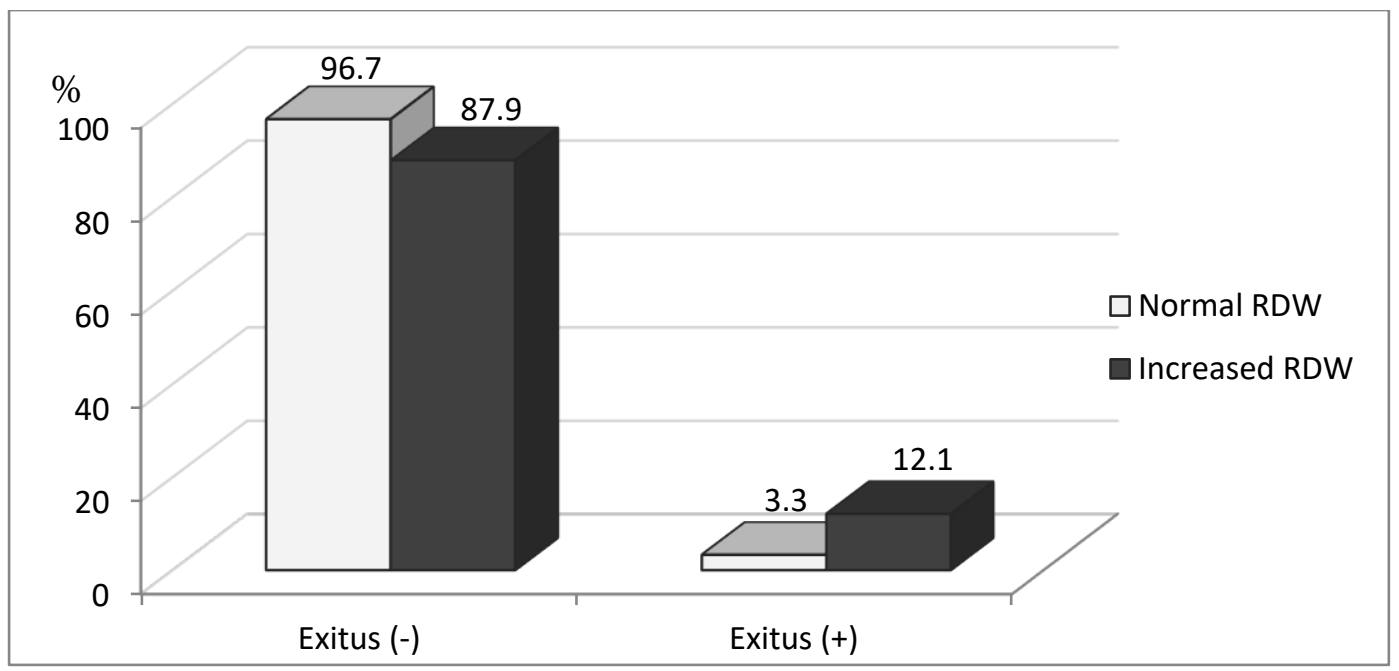

Figure: Comparison of RDW levels according to presence of exitus 